\title{
The Vulcan nerve pinch - cultural iconography anchors the proposal of a novel manual approach, the bow-string technique
}

\begin{abstract}
The Vulcan Nerve Pinch has a memorable and unique place in the cultural iconography of Star Trek. The proposed Bow-string technique uses this image to anchor the proposal of a novel technique preliminarily described here. The technique may possess both diagnostic and therapeutic implications for somatic dysfunction in the cervical region. It is based upon the established viscoelastic properties of collagen, in particular time-dependent stressrelaxation. The technique is reliant upon a high level of patient-centred engagement and intervenes at the contralateral side to a patient's active muscle effort. It is gentle, co-operative and runs for 2-4minutes. It theoretically engenders significant collaginous material change (lengthening) through the stress-relaxation characteristic of collagen associated with the imposition of a fixed mechanical strain. It is anticipated that the technique may possess a considerably greater persistence of effect when compared with shorter duration, repetitive passive stretch techniques, reliant on patient relaxation.
\end{abstract}

Volume 5 Issue 5 - 2017

M. C McGrath
University of Otago, Dunedin, New Zealand

Correspondence: M. C. McGrath, University of Otago, Dunedin, Country Practice Ltd. East Taieri, Mosgiel 9024, New Zealand,Email countrypracticeltd@gmail.com

Received: October 26, 2016 | Published: February 21, 2017

\section{Introduction}

According to a variety of sources the Vulcan Nerve Pinch (VNP) is a rapidly immobilising manual procedure usually performed by a Vulcan. ${ }^{1,2}$ While considered the province of science fiction it is nevertheless generally well known. It may therefore serve a useful purpose as an iconographic aide memoir, anchoring a novel manual technique that may have potential for both diagnostic and therapeutic utility.

The VNP is embodied by the application of manual force through digital pressure at a region located lateral to the junction of the head and neck of the target subject. While a similar location is replicated in the proposed and novel Bow-string technique, observation of the VNP suggests that the successful application of the technique does not appear to be reliant upon a highly specific hand-neck relationship. ${ }^{3}$ In this way and in contradistinction to the VNP the novel Bowstring technique will be shown to be reliant on specified anatomical placement.

There is a paucity of data regarding the formal testing of the both manual procedures. In the case of the VNP, empirical observation suggests that when the technique is applied by Vulcans it appears to lead to dependable and effective immobilization. ${ }^{3}$ In contrast, the delivery of the VNP by non-Vulcans suggests considerably diminished reliability and effectiveness. ${ }^{1}$ Formal in vivo testing of the VNP procedure by Vulcans on humans seems either unknown or undocumented. Whether data exists for in vivo testing between Vulcans also appears unknown as no formal record is identified at the Vulcan Academy of Science. ${ }^{5,6}$ In the case of human and animal testing, it is unlikely that the VNP will ever be formally tested and future ethical approval in either case seems unlikely given that the procedure has been described to cause the deleterious 'rupture of nerve fibers'. Nevertheless, a pragmatic example of the VNP technique applied to an animal, specifically a horse (equus ferus caballus) has been recorded. ${ }^{4}$ In this equine example, two key differences emerge when compared to the application of the VNP technique upon humanoids. First, the VNP technique was applied to the horse at a different location, namely to the dorsal caudal aspect of the equine cranium at its junction with the most cephalad point of the neck. Second, whereas in humanoids there appeared a rapid loss of consciousness, in the case of the horse the animal slowly adopted a recumbent position and did not appear to lose consciousness. ${ }^{4}$

Empirical observation of the multiple applications of the VNP technique under varying conditions provides substantive generic information, ${ }^{2}$ indicating widespread agreement that the junction of the base of the neck with the ipsilateral shoulder is a designated general point of application. However, the precise anatomical location of the VNP remains elusive. This has led to persisting uncertainty regarding the specific underlying anatomical structures likely to be implicated. Furthermore, whether such anatomical consideration is indeed necessary remains moot as it has been alleged that the putative effect may be entirely psionic. ${ }^{7}$ Indeed, it seems established that humans may not only lack sufficient manual strength but adequate psionic capability to attempt the procedure with any realistic expectation of reliable success. ${ }^{7}$

Irrespective of the precise location or of the alleged effect of the VNP technique in science fiction iconography, attention may nevertheless be drawn to its broad application and position of application and the relevant human anatomy of potential clinical significance. The aim of this commentary is to employ an association with cultural iconography to introduce a novel manual technique, referred to as the Bow-string technique.

\section{Regional anatomical considerations - a tale of two points}

Anatomical consideration of the region highlights its underlying complexity. The posterior triangle of the neck is formed embryonically from the division of a single large muscle mass into two muscles, the sternocleidomastoid and trapezius muscles. The posterior triangle is notionally formed within the anterior border of the middle portion of the trapezius muscle, the posterior border the sternocleidomastoid, and has as its base the middle third of the clavicle. ${ }^{8,9}$ The posterior triangle is superficial to and encompasses the deeper scalene triangle whose three boundaries are formed by the inner margins of the anterior and middle scalene muscles, and the superior border of the first rib. 
Ranney ${ }^{10}$ highlighted this region when anatomically redefining the 'thoracic outlet' as the 'cervico-axillary canal'. Two described landmarks or 'points' of key anatomical and clinical relevance lie within the boundaries of the posterior triangle, namely Erb's Point and the Punctum Nervosum. Of iconic interest is the observation that the latter point, the Punctum Nervosum does not appear coincident with the general application of the VNP, though the former point, Erb's Point, may more closely be approximated by it. Neither though could be associated with any attribution of the alleged instantaneous 'effect'.

\section{Erb's point}

Erb's Point ${ }^{11}$ is an eponymous point named by the eminent German neurologist Wilhelm Heinrich Erb, who described it in his 1883 text of electrotherapeutics as, 'a circumscribed point, about $2-3 \mathrm{~cm}$ above the clavicle, somewhat outside of the posterior border of the sternomastoid and immediately in front of the transverse process of the sixth cervical vertebra' Figure 1A,. ${ }^{12}$ The location of Erb's Point at the upper cervical outlet (nb. not to be confused with the cervical plexus), ${ }^{10}$ corresponding to the superior trunk of the brachial plexus (derived from the $\mathrm{C} 5$ and $\mathrm{C} 6$ roots), together with the suprascapular nerve and nerve to subclavius, and the bifurcation of the trunk into the posterior and lateral cords. This structural conglomeration may be visualised in Figure 1B. Erb termed this point of neurological electro-accessibility, the 'supraclavicular point', in which electrostimulation resulted in contraction of the 'deltoid, biceps, brachialis anticus, and supinator longus muscles'. The superficial accessibility of Erb's Point provides utility for electro-diagnostic motor conduction studies of the $\mathrm{C} 5$ and $\mathrm{C} 6$ roots potentially involving the lateral cord (musculocutaneous nerve - biceps, brachialis), the posterior cord (radial nerve - brachioradialis and axillary nerve - deltoid), and the suprascapular nerve and nerve to subclavius.

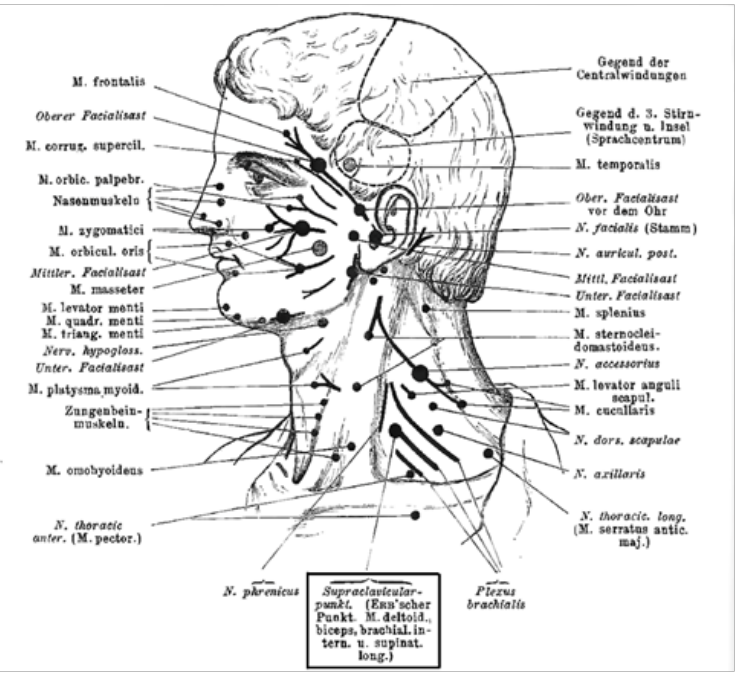

Figure IA Original depiction of ... the location of Erb's Point

\section{Punctum nervosum}

Over time, confusion appears to have arisen between the location of Erb's Point and another point located significantly more superiorly, referred to in Latin as the Punctum Nervosum or 'nerve point'. ${ }^{13,14}$ This latter point is associated with four cutaneous nerves that are the superficial branches of the ventral rami of $\mathrm{C} 2-\mathrm{C} 4$ and critically possess close spatial relations with prime moving muscles and fascia of the region. A schematic depiction of the location of the Punctum Nervosum and posterior cervical triangle is portrayed in Figure 2.

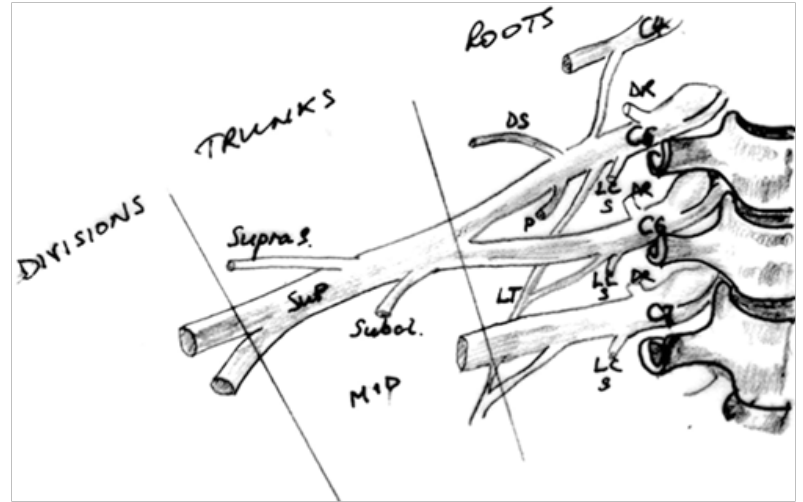

Figure IB Erb Point: brachial plexus anatomy schematic.

The most superficial location of a portion of the brachial plexus provides electro- accessibility of $\mathrm{C} 5$ and $\mathrm{C} 6$ roots, suprascapular nerve and nerve to subclavius. SupraS: suprascapular; Subcl: nerve to subclavius; SUP: superior trunk brachial plexus; MID: middle trunk brachial plexus; DS: dorsal scapular nerve; P: phrenic nerve; LT: long thoracic nerve; LC: nerve to longus colli; $\mathrm{S}$ : nerve to scalenes; DR: dorsal rami.

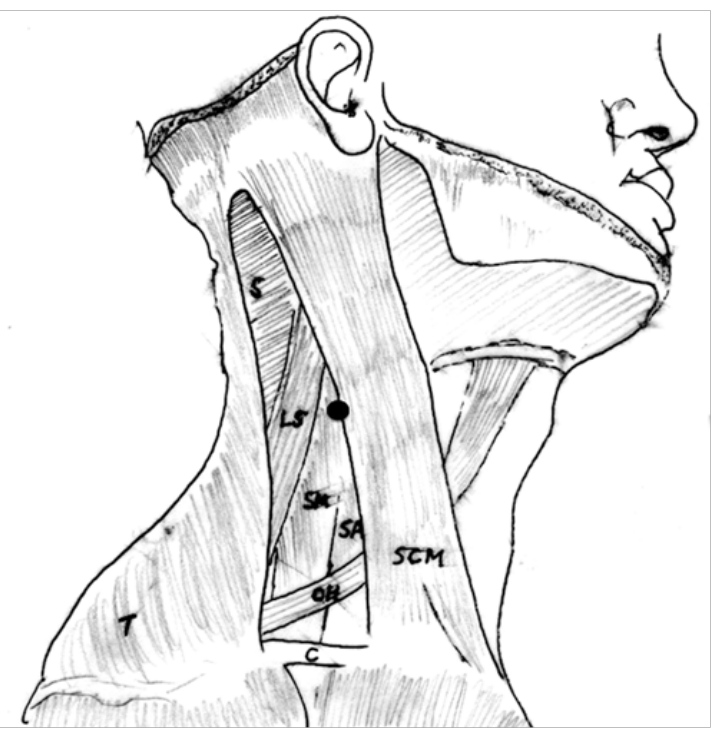

Figure 2 Location of the Punctum Nervosum and posterior triangle of the neck.

The posterior triangle of the neck is the triangle formed within the borders of the sternocleidomastoid muscle, trapezius muscle, and clavicle at the base. A black point demarcates the location of the Punctum Nervosum at the midpoint of the posterior border of the sternoscleidomastoid muscle (SCM).

T, trapezius; C, clavicle; S, splenius muscles; LS, levator scapula; SM, middle scalene; $\mathrm{SA}$, anterior scalene; $\mathrm{OH}$, omohyoid.

\section{Descriptive anatomy}

The Punctum Nervosum or 'nerve point' lies within the posterior cervical triangle and is located approximately halfway along the posterior border of the sternocledomastoid muscle at the approximate level of $\mathrm{C} 3{ }^{8,15}$ Four cutaneous branches splay out in a cruciform manner from the Punctum Nervosum. Two cutaneous branches divergently ascend (lesser occipital - C2, greater auricular-C2.C3), one cutaneous branch passes anteriorly (transverse cervical C2.C3 with an interconnection with the facial nerve (CN.VII) and one cutaneous branch descends giving rise to three further branches (supraclavicular C3.C4 -medial, intermediate and lateral branches) ${ }^{8,16}$ 
When considering the Punctum Nervosum and its four intimately associated cutaneous nerves, consider that each has moved from deeper levels between adjacent muscles, tracking toward their respective superficial cutaneous destinations, Figure 3.

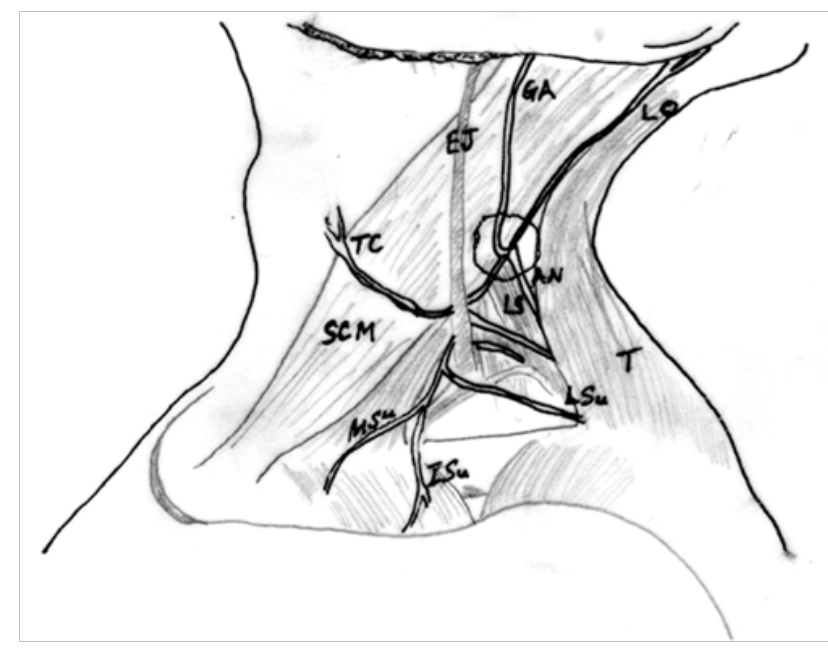

Figure 3 Sketch of the nerves of the Punctum Nervosum.

The Punctum Nervosum (centre, encircled) gives rise to four splaying cutaneous nerves, two ascending - the greater auricular nerve (GA), the lesser occipital nerve (LO); one anterior-the transverse cervical nerve (TC), coursing on the sternosceidomastoid (SCM) deep to the external jugular vein (E)), and one inferior - supraclavicular nerve, giving rise to three branches, medial (MSu), intermediate (ISu) and lateral (LSu). The muscles, trapezius (T) and levator scapular (LT) are identified, as is the accessory nerve (AN).

The ascending loops of the lesser occipital nerve and greater auricular nerve initially pass between the upper part of levator scapula and splenius capitis overlain by the sternocleidomastoid muscle, before looping around the posterior border of sternocleidomastoid. The lesser occipital nerve continues its ascent following the posterior border of sternocleidomastoid to arise through the deep fascia near the mastoid, becoming cutaneous and also linking with the greater occipital and greater auricular nerves. It variably supplies an approximate $2-3 \mathrm{~cm}$ area of cutaneous scalp that immediately surrounds the pinna of the ear, from the mastoid process posteriorly to a vertical line bordering the anterior margin of the pinna Figure 4.

The Punctum Nervosum (centre, encircled) gives rise to four splaying cutaneous nerves, two ascending - the greater auricular nerve (GA), the lesser occipital nerve (LO); one anterior-the transverse cervical nerve (TC), coursing on the sternosceidomastoid (SCM) deep to the external jugular vein (EJ), and one inferior - supraclavicular nerve, giving rise to three branches, medial (MSu), intermediate (ISu) and lateral $(\mathrm{LSu})$. The muscles, trapezius (T) and levator scapular (LT) are.

Cross-hatched shaded area depicts the approximate region supplied by the greater auricular nerve (GA); immediately posterior, the territorial region of the lesser occipital nerve (LO). More posterior still, toward the midline lies the territory of the greater occipital nerve (GO), and below, the dorsal rami C3 (DR); the transverse cervical nerve lies at the anterior neck (TC); below and behind this, lies the region innervated by the supraclavicular nerve and its branches. The territories of the fifth cranial nerve $(\mathrm{V})$, (trigeminal) and its branches, ophthalmic $(\mathrm{Op})$, maxillary $(\mathrm{Mx})$ and mandibular $(\mathrm{Mb})$ are also shown.

\section{Schematic depiction of the approximate cutaneous territories at the head and neck}

The greater auricular nerve emerges at the Punctum Nervosum and continues to loop superiorly as it ascends on the sternocleidomastoid, deep to platsyma with the external jugular vein to become cutaneous. ${ }^{8,15,16}$ At the level of the parotid gland, it divides into anterior and posterior branches. The former links with the facial nerve $(\mathrm{CN}$. VII) within the gland and is cutaneous over the parotid region. The latter is cutaneous over the mastoid and back of the ear, linking with the lesser occipital nerve, the auricular branch of the vagus (CN.X) and posterior auricular branch of the facial nerve (CN.VII) (Gray's Anatomy 2005). The variable territory covered includes much or all of the pinna, the region below the pinna that includes the lateral neck and adjacent angle of the mandible and may also include the region overlying and anterior to the temporomandibular joint Figure.4.

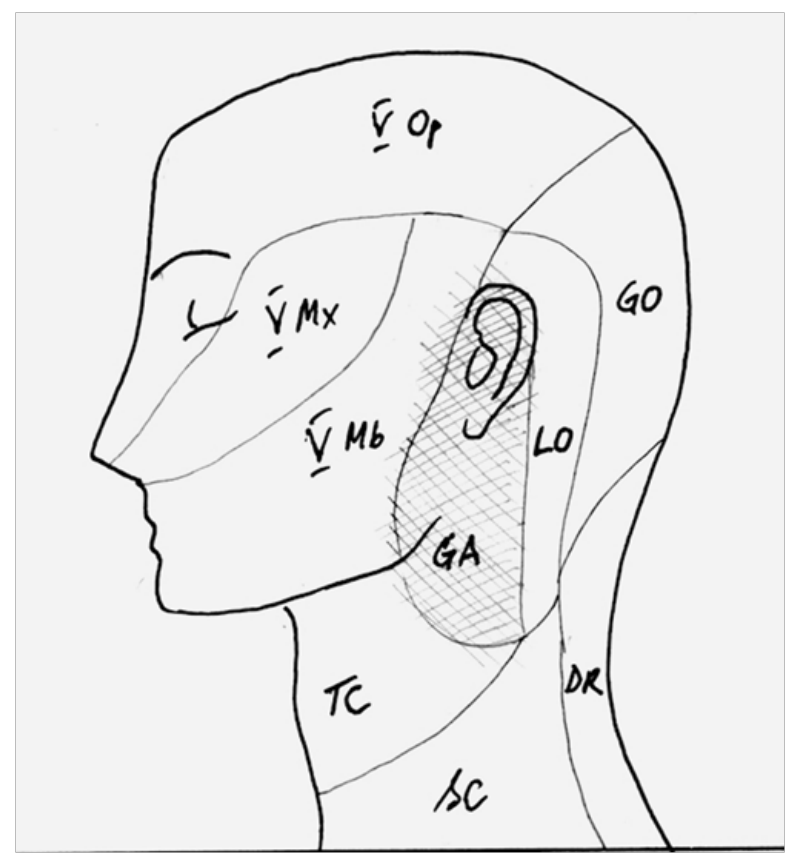

Figure 4 Schematic depicting cutaneous territories head and neck.

Cross-hatched shaded area depicts the approximate region supplied by the greater auricular nerve (GA); immediately posterior, the territorial region of the lesser occipital nerve (LO). More posterior still, toward the midline lies the territory of the greater occipital nerve (GO), and below, the dorsal rami C3 (DR); the transverse cervical nerve lies at the anterior neck (TC); below and behind this, lies the region innervated by the supraclavicular nerve and its branches. The territories of the fifth cranial nerve $(\mathrm{V})$, (trigeminal) and its branches, ophthalmic (Op), maxillary (Mx) and mandibular (Mb) are also shown.

The transverse cervical nerve also loops along a similar course though tracking more anteriorly, emerging at the Punctum Nervosum to pass anteriorly across the sternocleidomastoid deep to platysma to the anterior border of the muscle. Here it divides into ascending and descending branches, the former ascending to the submandibular region to link with the cervical branch of the facial nerve (Gray's Anatomy 205) and the latter, arising from platysma to be cutaneous, serving the anterolateral aspects of the neck down to the sternum Figure $4,{ }^{8}$

The supraclavicular nerve is the only superficial descending branch, emerging at the Punctum Nervosum to descend deep to platysma and to fan out distally at the clavicle into three branches, the 
medial, intermediate and lateral supraclavicular nerves. Collectively, a cutaneous region is served that spans the upper and posterior parts of the shoulder to the median plane anteriorly and the second rib inferiorly Figure 4.

Other well-known neurological structures of the region lie in relatively close proximity to the Punctum Nervosum. Moving in a lateral direction from the ventral midline these include the vagus nerve, the middle cervical sympathetic ganglion and the phrenic nerve. The vagus nerve and middle cervical sympathetic ganglion lie deep to and close to the common corotid artery and internal juguar vein. The phrenic nerve lies lateral to the internal jugular vein. Collectively, these lie to the front of the anterior scalene muscle at the level of C5 and C6.

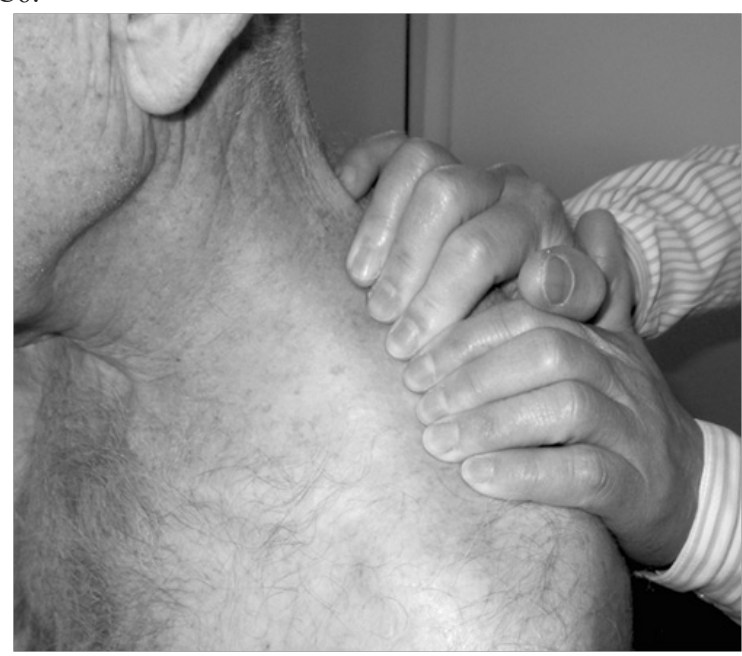

Figure 5 External clinician manual position and technique.

The clinician places both hands on one side of the neck of the patient, from the junction of the neck along the anterior border of the suprascapular aspect of the upper portion of the trapezius muscle, firmly emplacing the trapezius muscle with the fingers against the anterior border of this muscle.

\section{Manual intervention - potholes, pitfalls and fascia}

This preliminary report highlights anatomical considerations of the lateral neck and thoracic outlet with two potential issues in mind. First, diagnostic utility by clinical palpation under specified conditions for the presence of somatic dysfunction and second, potential treatment utility. From a diagnostic perspective the proposed Bow-string technique may help discern the nature of motion and tissue restriction in the frontal plain (lateroflexion) and transverse plane (axial). It remains a subject for further investigation to determine whether the relative contributions arising from articular, muscle and fascial structures may be reliably and validly discerned by observation and palpation alone.

When considering manual palpatory techniques it often appears that a clear separation between examination and treatment is not readily distinguishable either by an observer or by the patient, except by clinician intent and disclosure. The sensory confounding of both these elements is ubiquitous and cautionary. In the tactile milieu of manual palpation such procedures of examination and treatment are inevitably interdependent. Consider the omnipresent central influence of cortical input (cognition, perception, expectation) possessed by both the patient and by the clinician, together with the peripheral influence of manual pressure on low threshold afferent mechanoreceptors of touch, pressure and vibration and their associated wide dynamic range inter neurons in lamina IV and V at the dorsal horn. Such central and peripheral influences occur simultaneously in both parties at the point of manual engagement. ${ }^{17}$ The resultant sensory melange is a minefield for objectivity, a vital clinical quality that degrades rapidly in a proportional manner with both the time and the intensity of the manual clinical engagement.

In recent times, the functional and clinical anatomy of fascia in general and of the neck in particular has attracted growing attention from manual therapies. For example, there is a wider acceptance of the anatomical description of the 'Myodural Bridge' with its attendant clinical implications. ${ }^{18}$ Nevertheless, descriptions of regional cervical fascia remain challenged by inconsistencies of nomenclature. ${ }^{9}$ Fortunately, more recent work appears to have addressed this by providing a coherent description of this fascia. ${ }^{19}$ With the attribution of multiple physiological functions ${ }^{20}$ and their attendant clinical implications ${ }^{21-23}$ fascia has become a growing consideration in manual practice and its role in the Bow-string technique appears potentially significant.

\section{The Bow-string technique}

The biomechanical relevance of connective tissue: The biomechanical characteristics of fascia and muscle may have clinical relevance, particularly with respect to the 'feel' of these tissues and the relevance that this may have with respect to the presence or absence of somatic dysfunction. A biomechanical understanding of the basic constituent material, collagen becomes necessary.

When considering the 'feel' or 'texture' of 'myofascia' in general and fascia in particular, underlying processes of 'thickening', 'strainhardening' and fascial 'densification' have been described in addition to alterations of 'viscosity', 'fibrosis' and 'adhesion'. ${ }^{23,24}$ While such changes may remain notionally distinct from increases in muscle tonicity, which may better reflect and modulate neurologically based moment to moment changes in position and movement, how muscle and fascia may be distinguished and objectively evaluated solely by palpatory feel remains to be properly described and validated. It appears that sonographic imaging may also have a valuable investigative clinical role to play in this regard, as fascial thickening ${ }^{25,26}$ may be associated with myofascial pain states that a priori encompass states of somatic dysfunction with its accompanying aberrant state of muscle tonus and tissue textural change.

Using an experimental scenario that relies on collagen gel, a model of the mechanical behaviour of collagen protein in the body ${ }^{27}$ may potentially assist in the development of a rational quantification of the physical description (duration, applied stress and strain) of the proposed Bow-string technique. Shen and colleagues explored the stress-relaxation mechanisms in collagen matrices under the influence of cross-linking and hydration. Stress-relaxation is a mechanical characteristic of viscoelastic materials like collagen whereby a rapid fixed strain is imposed on the material and maintained. The stress in the material is seen to degrade over time as it undergoes viscoelastic deformation. (This process is distinct from 'creep' where a fixed stress is applied and maintained. The tissue undergoes subsequent viscoelastic deformation over time, lengthening in the process). It is frequently unclear or unstated in manual therapy whether one or other, or both these effects apply when techniques purporting to 'release' fascia or myofascia are undertaken.

Critically, Shen and colleagues identified three stress-relaxation peaks for collagen matrices at 0.3 second - 1second, 3seconds 1.5 minutes, and greater than 200seconds ( 3.5minutes). They 
hypothesized that these three relaxation peaks represent a hierarchy of collagen tissue stress-relaxation embodied in inter-fiber relaxation; inter-fibril relaxation, and fibril relaxation, respectively. This hierarchy appears consistent with the fact that fibril cross-linking appears the more durable property under long-term load, giving rise to the longest (slowest) fibril stress- relaxation time constant.

Citing the work of Yang et al., ${ }^{27}$ who identified two time constants for 'native' collagen, attributing these to inter-fibril and molecular sliding at approximately 1 second and 1 minute, compared with crosslinked collagen fibrils where they found 3 seconds and 250 seconds ( $\sim 4$ minutes), Shen and colleagues considered those results consistent with the range of time constants that they had identified for inter-and intra-fibril molecular sliding mechanisms. They also showed that the rate of stress-relaxation of collagen matrices increased linearly with the initial stress level, an observation consistent with the essential biomechanical properties of skin and tendon. The opposite relationship between stress level and stress-relaxation exists in ligamentous material, where the rate of stress-relaxation decreases with higher stress level, ligaments providing the requisite rigidity and stable tensile stress under load.

Pavan et al., ${ }^{21}$ cite the work of Stecco et al., ${ }^{29}$ highlighting that approximately $30 \%$ of the stress-relaxation of the deep fascia of the leg occurred within the first 2 minutes, a reduction in tissue stress that purportedly represents $90 \%$ of the stress-relaxation over an observation time of 4minutes. The implication here is that the lion's share of stress-relaxation may be theoretically achieved within 2 minutes from the onset of an applied fixed tissue strain. Two minutes may then be the relevant experimental consideration to import into the clinical setting, together with the idea of the application of a fixed strain.

More recently $\mathrm{Xu}$ et $\mathrm{al}^{30}{ }^{30}$ discussed the importance of the hydration levels of connective tissue and collagen in particular, highlighting the biphasic nature of collagen. They described how the solid phase is characterised by the collagen network and the liquid phase is characterised by the interstitial fluid, which may be tightly bound to collagen molecules or may be free. In the former, it stabilises the collagen molecules and leads to extensive interand intra- molecular hydrogen bonding. Together, these two phases facilitate the viscoelastic properties of collagen. More tightly packed collagen manifest in dehydration results in stronger and shorter intrafibril hydrogen bonding, that is, between disaccharide units made up of D-glucuronic acid and D-N-acetylglucosamine linked by $\beta 1.4$ and $\beta 1.3$ glycosidic linkages in addition to the inter- molecular hydrogen bonds between collagen fibrils. Such enhanced hydrogen bonding leads to mechanical stiffening of collagen, increasing its rigidity.

In the application then of manual techniques designed to purportedly 'release' fascia or 'myofascia' through the application of external therapeutic loads, whether applied across the skin or by axial or appendicular leverage, the time constants and initial tissue strain derived from the work of Shen et al., ${ }^{27}$ \& Yang et al., ${ }^{28}$ would appear to be an important biomaterial characteristic worthy of clinical consideration. As previously stated, 2 minutes may be the appropriate time over which to consider the application of a fixed strain to a myofascial structure.

\section{Introducing the bow-string technique - general description}

The Spock-like insight that resulted in the hypothesis of the Bowstring technique arose from the use of a standard palpatory examination of the cervical and thoracic outlet as part of the routine requirement to clinically evaluate the region. As the name (Bow-string) implies, it conceptualises the individual muscles of the region (upper portion of trapezius, levator scapular and the middle scalene) as 'bow-strings' and considers the utility of applying an additional external pivot to the 'string', much as the placement of an arrow and the subsequent draw by the archer creates an additional pivot point between the fixed points of a bow. Increasing strain (lengthening of the bow-string) engenders an increase of tensile stress in the bow-string. In considering the bow and arrow, the tensile stress of the string and elasticity of the bow serve to propel the arrow. In the case of the Bow-string technique, the tensile stress is developed in the ipsilateral resting myofascia of interest as the contralateral contraction is instigated by the patient leading to the implementation of contralateral axial rotation or lateral flexion, which tightens the ipslateral myofascial structures against the impinging external pivot provided by the clinician. The clinician may judge the rate of stress generation within the myofascial tissue undergoing increasing strain and simultaneously observe the commensurate degree of contralateral motion.

Further consideration of this potential technique suggested that a more sustained application of the Bow-string technique may possess a potential to modulate the resting length of regional myofascia through the stress-relaxation characteristic of affected biomaterials (collagen) previously described thereby potentially influencing regional somatic dysfunction.

It is emphasised that any de novo expression of this technique whether in manual testing or in the quest of potential therapeutic utility is bereft of data attesting to reliability and validity both in putative manual assessment and in any potential for treatment utility. The prima facie indication is a requirement for the modulation of regional somatic dysfunction. Thus far the Bowstring technique is descriptive and anecdotal. Use of this manual technique either in evaluation or in therapeutic intervention could be considered experimental and therefore may be subject to ethics approval. Relative and absolute contraindications may theoretically apply in a clinical setting with specific consideration accorded to regional neurovascular and lymphatic structures and their potential for anatomical variation.

\section{The Bow-string technique - specific considerations}

As described, the Bow-string technique relies on the application of an external fixed pivot (clinician's palpating fingers) placed firmly between the origin and insertion of a muscle (upper portion of trapezius, levator scapular, middle scalene) in a manner that takes-up or reduces the 'slack' in the muscle until a state of moderate resistance may be felt. This is the first step in creating an increase of material strain within the muscle. The patient is then requested to steadily move the region in a manner that lengthens the distance between the origin and insertion of the ipsilateral myofascia under a direct external imposition of an external digital pivot. Importantly, this may occur when the patient contracts the contralateral muscles. The pressure of the manually applied external pivot on the relaxed ipsilateral side should be maintained continuously as the contralateral movement occurs. The clinician may feel the reaction force of the underlying myofascia increase as the mechanical strain (lengthening) within the soft-tissue escalates. The patient continues with the movement unless excessive tenderness or pain supervenes until a point is reached when the reaction force against the clinician's external firm grip approaches the limit of what may comfortably and readily be constrained by the clinician. At this point, a fixed strain is considered to be applied and critically, neither the clinician nor patient should alter the mechanical status quo, particular if the technique is being employed from a therapeutic rather than diagnostic perspective. 
When used as a diagnostic tool, the assessment of contralateral movement with its pro rata developing ipsilateral tension (felt under the fingers of the clinican) with or without pain may be easily evaluated. When employed as a possible therapy, the point of maximum strain should be maintained for a period of not less than 2 minutes and not longer than 4 minutes. The former period of 2 minutes is coincident with the acquisition of a 'lions share' of the stress-relaxation of local collagen discussed previously, or in the case of the latter, encompassing all the peaks of stress-relaxation associated with collagen.

\section{Further technique specifics}

Emplacement of the clinician's fingers against the ventral border of the suprascapular aspect of the upper portion of trapezius of the seated or standing patient. The clinician grips firmly, preventing the muscle from bow-stringing with subsequent contralateral axial or lateral movement of a patient's the neck, when the patient is requested to turn their head away, or to laterally flex their head and neck away from the side of contact, depending on the myofascial structures engaged by the clinician - trapezius, levator scapular, anterior / middle scalene.

The clinician 'constrains' the muscle while the patient applies as much turning force in a manner proportionate to the mechanical strain (and potential discomfort) felt under the clinician's constraining fingers. Once a peak tolerable point reached, the patient moves no further. The clinician's maintenance of a constant grip that does not vary from the start of the manoeuvre appears critical to the theoretical success of the technique. The resultant degree of ipsilateral muscle strain and the speed with which it develops in association with the contralateral movement may help to indicate regional tissue change. Diagnostically, the position of strain may be held by the patient briefly (a few seconds) in order to help establish a diagnostic clinical impression. The patient 'controls' the manoeuvre based upon their perceived discomfort, and where necessary may retreat from and advise the clinician of the presence of discomfort.

The clinician's examining fingers may next be emplaced anteriorly and a little deeper, encountering the levator scapular muscle. A similar technique is reapplied, except in the case of this muscle the motion requested of the patient is lateral flexion away from the clinician. The clinician's fingers may be moved further again to the middle scalene. While gently restraining this myofascial structure avoiding unnecessary or direct pressure on the nearby adjacent neurovascular structures, the patient once again turns their neck in contralateral axial rotation away from the side of the external pivot. The relaxed ipsilateral levator scapula muscle may be constrained from bowstringing.

As described, in each instance and on each side this putatively diagnostic manual technique may be modified toward potential therapeutic intervention by the increase of time spent in the occupation of a fixed strain loading. Accordingly, the time constraints discussed previously associated with a theoretical duration of application required to induce collaginous stress- relaxation.

The clinician places both hands on one side of the neck of the patient, from the junction of the neck along the anterior border of the suprascapular aspect of the upper portion of the trapezius muscle, firmly emplacing the trapezius muscle with the fingers against the anterior border of this muscle.

\section{Discussion}

The cultural iconography of Star Trek and the Vulcan Nerve Pinch spans Generations. It may provide a helpful association for the introduction of a novel technique, the Bow-string technique based on the therapeutic position of the intervening clinician in relation to a patient, in addition to the clinician hand placement. This novel technique relies on an understanding of the underlying time constants associated with the visco-elastic property of stress-relaxation that has been shown to occur in collaginous soft-tissue exposed to a fixed strain. The subsequent stress- relaxation that may occur in the prestrained collaginous tissue does so over known time constants where, sustained over a period of two minutes it may be considered to capture the 'lions share' of the viscoelastic yielding effect.

Most involved in manual therapy may readily attest to the frequent clinical finding of an aberrant state of muscle tension in the neckshoulder region, whether at the 'headache' or 'outlet' aspects of the thorax. The simplest of literature searches establishes a list of potential underlying causes of headache that is truly a litany of tribulation. The role of the trigemino-cervical reflex is well established ${ }^{31,32}$ with the literature reporting that as much as $47 \%$ of the global population suffer with headache of which some $15-20 \%$ are described as cervicogenic. ${ }^{33}$ Thoracic outlet syndrome and in particular its variant of neurological thoracic outlet syndrome presents yet another clinical syndrome whose basis can frequently be related to or associated with sustained aberrant patterns of muscle tonicity, with manual therapy and management providing a first line of intervention.

The Punctum Nervosum may possess clinical relevance when considering the Bow-string technique for in considering the application of the Bow-string technique and the imposition of tissue strain upon the muscles adjacent to and surrounding the cutaneous nerves of the cervical plexus, a theoretical impingement may arise between the two, potentially leading to referred dysthesia or pain projected over the cutaneous distribution. The greater auricular nerve and the lesser occipital nerve, with or without facial nerve involvement would appear to be potential candidates for this effect. It is anticipated that this may potentially occur as these cutaneous nerves undergo a mild regional neuropraxia, consequent to the pinching myofascia of the upper part of levator scapula and splenius capitis overlain by the sternocleidomastoid muscle. It may conceivably also be more likely to occur when the pre-existing state of myofascia in the region could be clinically considered a priori as 'shortened' or 'tight'. A number of additional potential explanations may exist, such as the variation of course taken by the cutaneous nerves in single individuals, which might encompass a description of normal anatomical variation. Further morphological investigation is required.

Of interest to those involved in manual care are palpable and discernible patterns of amplified tonicity of the myofascia of the cervical region, which includes the anatomy of the sub-occipital, cervical and thoracic-outlet. In the first instance, the Bow-string technique offers another way in which regional tonicity and sensitivity may be evaluated, and in the second, creates a potential opportunity for therapeutic intervention and potential modification. There is a further added advantage, namely that the technique may be considered highly patient centred, being nearly entirely dependent on the patient for the generation of mechanical strain through the implementation of contra-lateral muscle contraction. The clinician merely imposes a steady, firm external pivot point on the relaxed ipsilateral side in order to pre-strain a relaxed myofascial structure. The patient generates the changing state that may only occur once they move the origin and insertion of their relaxed muscle apart under the controlled and active engagement of their contralateral muscles. ${ }^{34-36}$

\section{Potential positional limitations}

The technique appears simple to apply, requiring of minimal force and should be conducted with the patient in a seated or upright 
position. The technique is impractical in a supine position as substantial modification to head, neck, scapulocostal, and scapulothoracic relations develops because of a significant reduction in axial column load and change in position. This occurs when the muscle reaction forces required to control the mass of the cranium, maintain neck posture and control movement are provided instead by the surface of an examination table. It appears very difficult or impossible to create sufficient mechanical strain in the designated myofascia, with the patient in a supine or prone position.

\section{Acknowledgments}

None.

\section{Conflicts of interest}

Author declares there are no conflicts of interest.

\section{Funding}

None.

\section{References}

1. http://memory-alpha.wikia.com/wiki/Vulcan_nerve_pinch

2. https://en.wikipedia.org/wiki/Vulcan_nerve_pinch\#Physiology

3. https://www.youtube.com/watch?v=TgMjV3Yse3U

4. https://www.youtube.com/watch?v=VYezfpJvFsw

5. http://memory-beta.wikia.com/wiki/Main_Page

6. http://memory-alpha.wikia.com/wiki/Vulcan Science Academy

7. https://www.quora.com/How-do-you-properly-perform-a-Vulcannerve-pinch-on-someone

8. Gray, Henry. Gray's Anatomy 2005 The Anatomical Basis of Clinical Practice. (39th edn), Elsevier Churchill Livingstone, Edinburgh, Scotland. 2005.pp.534.

9. Guidera AK, Dawes PJD, Stringer MD . Cervical fascia: a terminological pain in the neck. ANZ J Surg. 2012;82(11):786-791.

10. Ranney D. Thoracic outlet: An anatomical redefinition that makes clinical sense. Clin Anat. 1996;9(1):56-52.

11. Tubbs RS, Loukas M, Salter GE, et al. Wilhelm Erb and Erb's Point Clin Anat. 2007;20(5):486-488.

12. Erb W. Handbook of electro-therapeutics 1883. In: Putzel L, Editor. New Jersey, USA: William Wood and Company; x 1883. pp. 122-124.

13. Carswell AJ, Clamp PJ, Titoria P, et al. Erb's Point - Do ear, nose and throat surgeons know where it is? - Letter to the Editor. Clinical Anatomy. 2010;23(1):127.

14. Tubbs RS, Loukas M. Errare humanum est perservare diabolicum Letter to the Editor. Clin Anat. 2010;23:128.

15. Netter FH. Atlas of Human Anatomy. 3rd edn. New Jersey, USA Teterboro; 2003. pp. 123-124.

16. http://www.apicareonline.com/the-cervical-plexus-anatomy-andultrasound-guided-blocks/

17. McGrath MC . Palpation of the sacroiliac joint: an anatomical and sensory challenge. International Journal of Osteopathic Medicine. 2006;9(3):103-107.
18. Enix DE, Scali F, Pontell ME. The cervical myodural bridge, a review of literature and clinical implications. J Can Chiropr Assoc. 2014;58(2): 184-192.

19. Guidera AK, Dawes PJD, Fong A, et al. Head and neck fascia and compartments: No space for spaces. Wiley Online Library. Head Neck. 2014;36(7):1058-1068.

20. Swanson RL. Biotensegrity: A unifying theory of biological architecture with applications to osteopathic practice. Education, and Research - A Review and Analysis. J Am Osteopath Assoc. 2013;113(1):34-52.

21. Pavan PG, Stecco A, Stern R, et al. Painful connections: densification versus fibrosis of fascia. Curr Pain Headache Rep. 2014; 18(8):441419

22. Klingler W, Velders M, Hoppe K, et al. Clinical relevance of fascial tissue and dysfunctions. Curr Pain Headache Rep. 2014;18(8):439-446.

23. Kumka M, Bonar J. Fascia: a morphological description and classification system based on a literature review. J Can Chiropr Assoc. 2012;56(3):179- 191 .

24. Stecco A, Gesi M, Stecco C, et al. Fascial components of the myofascial pain syndrome. Curr Pain Headache Rep. 2013;17(8):352.

25. Stecco A, Meneghini A, Stern R, et al. Ultrasonography in myofascial neck pain: randomized clinical trial for diagnosis and follow-up. Surg Radiol Anat. 2014;36(3):243-253.

26. Harley JJ. Reliability of deep cervical fascia and sternocleidomastoid thickness measurements using ultrasound imaging. Master of Osteopathy thesis, Unitec Institute of Technology. 2016;1-89.

27. Shen ZL, Kahn H, Ballarini R, et al. Viscoelastic properties of isolated collagen fibrils. Biophys Journal. 2011;100(12):3008-3015.

28. Yang L, van der Werf KO, Koopman BFJM, et al. Micromechanical bending of single collagen fibrils using atomic force microscopy. $J$ Biomed Mater Res A. 2007;82(1):160-168.

29. Stecco C, Pavan P, Pachera P, et al. Investigation of the mechanical properties of the human crural fascia and their possible clinica implications. urg Radiol Anat. 2014;36(1):25-32.

30. $\mathrm{Xu} \mathrm{B}, \mathrm{Li} \mathrm{H}$, Zhang Y. Understanding the viscoelastic behavior of collagen matrices through relaxation time distribution spectrum. Biomatter. 2013;3(3):e24651.

31. Bogduk N. The anatomy and physiology of headache. Biomed Pharmacother. 1995;49(10):435-445.

32. Fernandez-de-Las-Penas C . Myofascial head pain. Current Pain and Headache Reports. 2015;19:28.

33. Page P. Cervicogenic headaches: an evidence-led approach to clinical management. International Journal of Sports Physical Therapy. $2011 ; 6: 254-266$

34. Fisher DA. A simple method of identifying the spinal accessory nerve. Dermatologic Surgery: official publication for American Society for Dermatologic Surgey. 2000;26:384-386.

35. Eisele DW. Head and Neck Fascia and Compartments. Head Neck Clinical review. 2014;1058-1068.

36. Kuhn JE, Lebus GF, Bible JE. Thoracic outlet syndrome. Review Article. J Am Acad Orthop Surg. 2015;23(4):222-232. 